\title{
Improved Intuitive Automated Attendance System using Unorthodox Algorithms
}

\author{
Prashant Wakchaure \\ Department of Computer Engineering \\ Sinhgad Academy of Engineering, \\ Kondhwa (Bk.), Pune 411048, Maharashtra, India \\ Kaushik Rohida \\ Department of Computer Engineering \\ Sinhgad Academy of Engineering, \\ Kondhwa (Bk.), Pune 411048, Maharashtra, India
}

\author{
Saad Shaikh \\ Department of Computer Engineering \\ Sinhgad Academy of Engineering, \\ Kondhwa (Bk.), Pune 411048, Maharashtra, India \\ Sachita Singh \\ Department of Computer Engineering \\ Sinhgad Academy of Engineering, \\ Kondhwa (Bk.), Pune 411048, Maharashtra, India
}

\author{
Ashish Ramdasi \\ Department of Computer Engineering \\ Sinhgad Academy of Engineering, \\ Kondhwa (Bk.), Pune 411048, Maharashtra, India
}

\begin{abstract}
One of the troublesome undertakings in Image Processing is accurately recognizing faces in videos, which consecutively offers less precision. Recognizing faces irrespective of the gender is an effortless task for humans; however, from a machine or a robot's perspective, it is an entangled task. Identifying the gender of an individual using their voice is simple when contrasted with facial pictures. This kind of binary classification can be used in various applications, for example, a surveillance system, directed publicizing, demographic gathering, human-machine interaction, content-based indexing and searching, biometrics, and so forth. In order to detect faces with ranging accuracy, there are multiple methods proposed by researchers, yet no existing system can produce an accuracy of $100 \%$. In this manuscript, an automated attendance system is built, to recognize faces from existing trained dataset of student images collected from live training. Along with Face Recognition, the system is equipped with second-level security of QR code scanning. At the testing stage, the experimental results exhibited a precision of $93.00 \%$ and an f1-score of 0.9 .
\end{abstract}

\section{Keywords}

Face Recognition, Images, Haar-feature classifier, face detection, Support Vector Machine, attendance, QR Code, f1-score, Local binary Pattern

\section{INTRODUCTION}

Efficient implementation of face detection and recognition algorithm leads to a successful outcome of an automated attendance system. Face recognition has highlighted specialists in the fields of security and access, signal processing, advertising, and health care. Face identification is extensively utilized in a plethora of applications, for example, recognition of VIPs at functional events, recognizing the owner in a self-driving car, entryway control framework and so on. It has additionally shown accommodation in handling multimedia data preparing zones. Therefore, one of the significant application areas under Image Processing is student attendance system.

As authentication is a significant issue in PC based correspondence, the use of facial recognition is dubious. Then again, it is intuitive to use and more systematized according to the requirements. Subsequently, this framework has been actualized by taking uncomplicated attendance marking into consideration by using unorthodox algorithms for face detection and recognition.

The manual work of the person identification and marking the attendance is an exceptionally convoluted and tedious assignment. Nevertheless, it is obligatory in schools and colleges of India. The manual attendance is over the long haul submitted to the office on a month to month premise to oversee. Being non-automatic, if there happens to be a mistake in the marking of attendance, the scholar below 75\% might be impeded from sitting for the examinations. In such a circumstance, there must be a quicker and progressively effective method for chronicling it [16]. One mainstream technique is the biometric scanner [18]. Even though these methodologies can be accurately designed, but simultaneously can be moreover tedious indistinguishable to manual attendance. Different techniques, for example, identity card scanners likewise have a similar issue [17]. The odds of the attendance intermediaries are more in manual attendance system. Existing automated systems overcome these difficulties, but have drawbacks like cost, counterfeit attendance, exactness, and so forth. So there is a need to implement a secure attendance system which avoids all the above problems; face recognition can be an ultimate solution for simple automated 


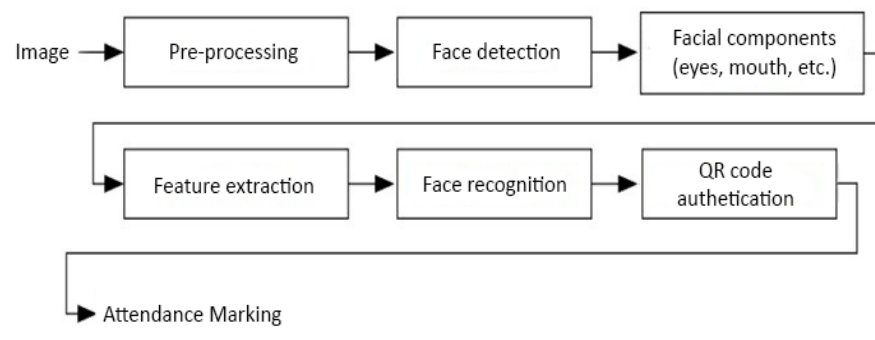

Fig. 1. Basic flow of Attendance Marking

attendance marking.

The system presented in this paper is an intuitive automated face recognition attendance system which has its source in the powerful Raspberry Pi 3 Model B+. It will provide the facial features that are extracted from the face images for the classification of person. Also there will be dual security in the disguise of QR code scanner once the authorized face is recognized by the system. The basic flow of the system is given in Figure 1

Basically, the live images are captured initially and then processing them progressively, the result set is produced for automatic student's attendance marking system. Facial Recognition is a procedure performed by a capable system, which comprises:

(1) Finding faces in the pictures; otherwise called face detection.

(2) Facial feature extraction from the recognized face area.

(3) Examining the development of facial attributes along with the adjustments made within the aspects.

(4) Classifying this information for detecting the face of particular students.

In the coming sections, following topics are discussed: Related works are shown in Section 2 Section 3 shows the working and implementation of our proposed system, Section 4 shows the experimental results, Section 5 discusses the future scope and in the final section some concluding remarks are made.

\section{RELATED WORK}

We researched all the existing techniques for face recognition and detection in the previous paper [20]. Several works proposed various methods to determine facial pattern changes, face detection, face recognition, and development related to the maturing procedure, both from mental and organic perspectives. A portion of these works was even helpful in the assurance of proper facial highlights for face acknowledgment. Age estimation is a functioning subject in the present time considering the developing need of getting necessities or inclinations in various parts of the everyday life of an individual. Hamdi Dibeklioglu et al. proposes a system [1] which shows that other than appearance data we can use facial elements in age estimation. They propose a strategy to concentrate and use dynamic highlights for age estimation utilizing an individual's grin. They showed that unconstrained and presented grins have extraordinary and unmistakable elements and spontaneity specific age estimation essentially outflanks the general methodology. Using spontaneity data diminishes the mean absolute error by up to $21 \%$ in assessing age dependent on appearance.

Yang Zhong et al. proposes the methodology of predicting face attributes using trained CNN?s [2] for face recognition. Consol- idating with customary face localization strategies, they use offthe-shelf architectures trained for face recognition to fabricate facial descriptors. Their investigations present the correspondence assorted variety between the best performing portrayals and the describable human qualities. They additionally uncover that the intermediate portrayals from CNN?s are viable in foreseeing facial traits. They accomplished exact attribute prediction using the two datasets LFWA and CelebA, by appropriately leveraging the offthe-shelf CNN representations.

Max Ehrlich et al. presents [3] the facial trait classification using a multiple task study approach. Numerous trait stratification for faces uses shared portion of feature representation. The mutual representation uses an iterative learning approach comprising a bottom-up/top-down pass; that is the Restricted Boltzmann Machine (RBM) based model, improved with a considered multiple tasks segment to evolve into MT-RBM model. The Celebrity Faces (CelebA), the Multi-task Facial Landmarks (MTFL), and the ChaLearn challenge dataset are utilized to give the performance.

Jianlong Fu et al. [4] presents intermittent attention CNN for finegrained acknowledgment, which adapts recursively discriminative area consideration and locale based element portrayal at numerous scales. They use Intra-scale categorization loss and Inter-scale classification loss to optimize the proposed RA-CNN. It is done so as to commonly learn precise area consideration and fine-grained portrayal which potentially increases the accuracy by $3.7 \%, 3.8 \%$, $3.3 \%$, on Stanford Dogs, Cars and CUB Birds, respectively.

The straightforward and completely programmed panoramic picture based pose-invariant face acknowledgment technique [5]; presented by Yun-Fu Liu et al. which gives the face image captured at an arbitrary angle within 45 in yaw and 22.5 in pitch during identification to give an excellent accuracy with low complexity. The confined transforming treatment is utilized to manage the majority of the conceivable geometric twisting issues in the recognition phase.

In this paper [6] presented by Xiang-Yu Li et al., the face is recognized using (HOG) feature extraction and quick principal component analysis algorithm. They used the Haar-feature classifier for the native information. Moreover, the HOG highlights are pulled back from the given picture information and the PCA measurement reduction is handled, and the (SVM) algorithm is utilized to discern the face.

The paper [7] presented by Mashhood Sajid et al. gives an applied model for a robotized attendance framework by using an approval procedure which is integral and fundamental and improves the unwavering quality of the model. They used facial fiducial points of the understudy's countenances through which attendance can be registered.

S Poornima et al. [8] gives a framework that can consequently distinguish the student in the classroom imprints the participation by perceiving their countenances alongside sex characterization and sound yield. This system is developed by capturing ongoing human faces in the class. The appearances recognized are coordinated against the reference ones in the dataset, and then their attendance is marked. At last, the non-attendant records are spoken out loud through a voice output framework for affirmation. Furthermore, the framework is trained to classify the sexual orientation of the students present in the class.

Hemantkumar Rathod et al. [9] proposed an attendance management framework which appraises the participation of every understudy by constant clicking of pictures for quite a while and finding the best-restricted picture for processing. This method is secure, dependable and simple to use. They executed the general framework in MATLAB, henceforth it does not merit the thought. 
Yueqi Duan et al. [10] gives a context-aware local binary feature learning (CA-LBFL) technique for face acknowledgment. The principle highlight of CA-LBFL is that it exploits the logical data of adjoining bits by obliging the number of movements from different double bits so that in the long run extra strong data can be exploited for face portrayal. It likewise gives two techniques to miscellaneous face coordinating by coupled learning strategies (C-CA-LBFL and C-CA-LBMFL).

Samuel Lukas et al. [11] propose a strategy for a student attendance framework using a face acknowledgment system by consolidating Discrete Wavelet Transforms (DWT) and Discrete Cosine Transform (DCT). The highlights of understudy's face are thereby extracted and by applying the Radial Basis Function (RBF), facial articles are categorized.

The paper [12] introduced by Manop Phankokkruad et al. compares facial recognition accuracy of three conventional algorithms specifically Eigenfaces, LBPH and Fisherfaces. The accuracy obtained from LBPH is $81.67 \%$ off in still-image-based testing. In this way, LBPH is the most reasonable algorithm to show signs of improvement in accuracy.

The methodology [13] presented by Albert Ali Salah et al. consolidates appearance highlights with outward appearance elements. The technique concludes that the input video starts with a slight frontal face, and has the whole length of a grin (or nauseate) appearance. For grin and appall articulation in proposed framework they use UvA-NEMO Smile Database. The modification in elements of grins for various ages is broke down.

Ziwei Liu et al. [14] has proposed a novel and profound learning system for face trait prediction in the wild. Their method is robust to foundation clutters and face varieties. They have likewise uncovered many significant realities about learning face portrayal, which shed a light on new headings of face limitation and portrayal learning.

A face detection strategy under complex illumination that consolidates the skin shading division and cost-touchy Adaboost calculation is proposed in this paper [15]. In the first place, by utilizing the characteristic of human skin shading grouping in the color space, the skin shading region in $\mathrm{YCbCr}$ color space is extricated and various unimportant foundations are rejected; at that point for helping the inadequacies of the Adaboost calculation, they bring the costtouchy capacity into the Adaboost computation; finally the shading division and cost-delicate Adaboost computation are joined for the face discovery. Trial results exhibit that the proposed identification strategy has a higher location rate and recognition speed, which can quickly adjust to the real field environment.

In [16], Raghav Apoorv and Puja Mathur aim at implementing a low vitality Bluetooth based attendance management framework. It makes use of beacons using Bluetooth Low Energy that communicates with an android application. They use the application to gather the information from the sensors and store it as per the dates. This helps the administrator to record and investigate the participation of all students. They have also introduced other techniques for maintaining a strategic distance from intermediaries or false attendances.

\section{PROPOSED SYSTEM}

\subsection{System Architecture}

User uploads a video/grabs images using a live camera on the application, the application then extracts frames from the video. These frames are saved on the local machine. Frames are usually $640 \times 480$ formats. Haar cascade classifier is applied on images for detecting the faces. Once the faces are obtained, preprocessing on the images takes place, which include noise removal, normalization, etc. After applying pre-processing, the result is then matched with the face which is saved in the database. Once the face is matched, the students have to scan their unique $\mathrm{QR}$ code. The unique $\mathrm{QR}$ code is allocated to every student at the time of registration. Once the QR code is scanned the attendance is marked of the particular student in the database.

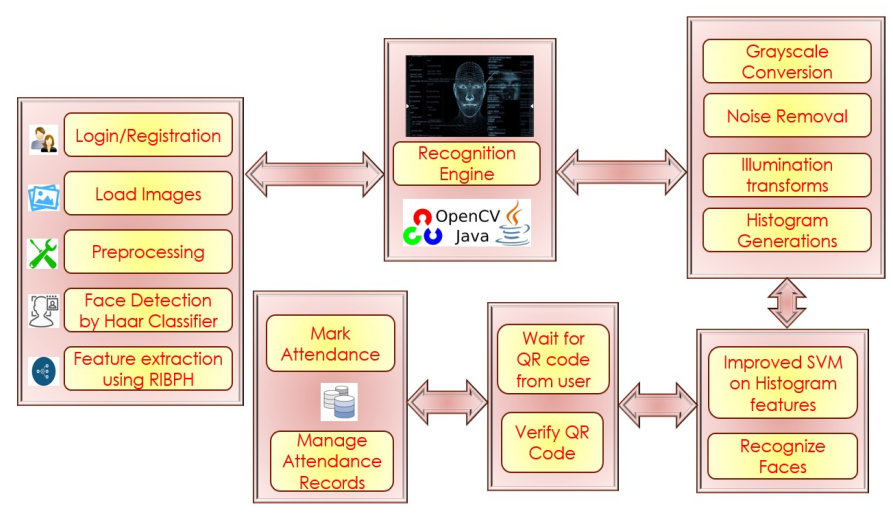

Fig. 2. Architecture Diagram

(1) RGB to Gray Scale Image:

(a) Convert the image into Gray-scale by taking the average of each pixel RGB.

(2) Local Binary Patterns Histograms:

(a) The examined window is divided into the cells (e.g. 16×16 pixels for each cell). Juxtapose the pixel to each of its 8 neighbors for every pixel in the cell. After the above process go around with the pixel in a circular format, i.e. clockwise or counter-clockwise.

(b) Compare the center pixel's value with the neighbor's, and if the value is greater than write " 0 " otherwise " 1 ". After that, the binary number ( 8 digits) is changed into decimal form for convenience.

(c) Based on the frequency of each integer the histogram is evaluated. The evaluated histogram is of the size of the 256-dimensional feature vector.

i. The histogram is then optionally normalized.

ii. Concatenate the (normalized) histograms of all cells. This gives a feature vector for the entire window.

(d) The feature set is then saved to a model for later matching process.

(e) Using SVC or Support Vector Classification algorithm analysis on the LBP facial features, detect the face of the user and mark his/her attendance.

\subsection{System Flowchart}




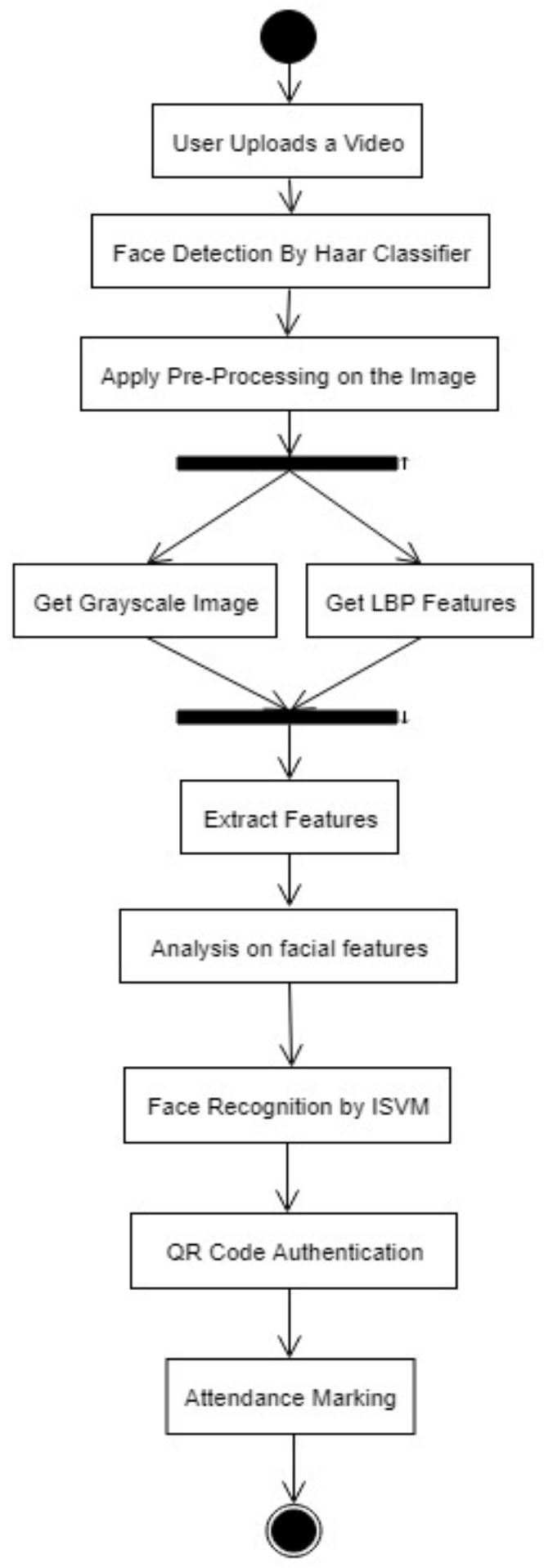

Fig. 3. System Flowchart

\subsection{Haar Cascade Classifier}

Paul Viola and Michael Jones proposed an innovative method called Haar cascade classifier which is used for object detection. This method is similar to machine learning in which a cascade function is trained with the help of positive and negative images. It is then used for detecting objects in other images. This classifier is mainly used to detect faces and body parts in an image but it can also be trained to identify any object in the image. This classifier is trained with the help of many positive imaged with faces and negative images. Then we need to extract features from it. Haar features are selected in the first step. Haar features basically consider adjacent rectangles at some specific locations inside a detection window, then add up the intensities of the pixels in each region and finally calculate the difference between these sums. Most of the features are irrelevant. AdaBoost is used to select the best features. Haar feature is only a "weak classifier", so a large number of Haar features are required to describe an object with sufficient accuracy and so we organize them into cascade classifiers to form a strong classifier.

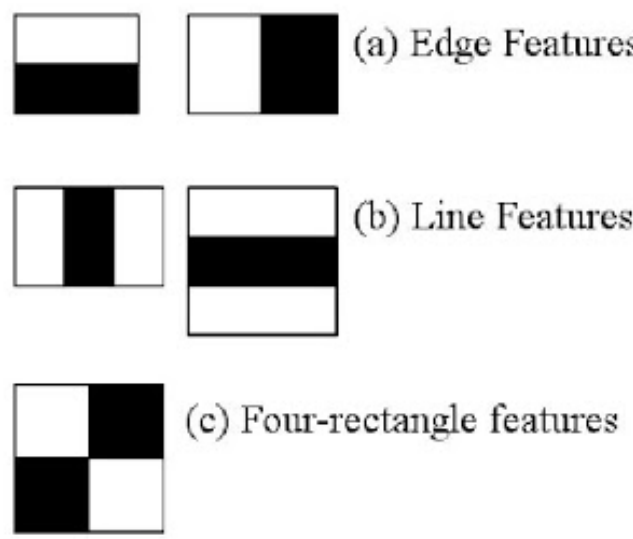

Fig. 4. Haar Features

\subsection{LBP Histograms}

Local Binary Pattern(LBP) is an extremely simple operator which assigns labels to the pixels by the use of application of thresholds to the neighbors of every pixel and assumes the output as a binary number. LBPH uses 4 parameters:

(1) Radius: used to construct the circular LBP and represent the radius around the central pixel. It is usually set to 1 .

(2) Neighbors: sample points to create the orbicular local binary pattern.

(3) Grid X: the number of cells in the horizontal direction.

(4) Grid Y: the number of cells in the vertical direction.

First the intermediate image is formed which describes the better version of the original image, by highlighting the facial features. Sliding window concept is used by the algorithm which is based on parameters and radius. Figure 5 shows this procedure:

Now, the generated image is divided into multiple grids with the help of Grid X and Grid Y. 

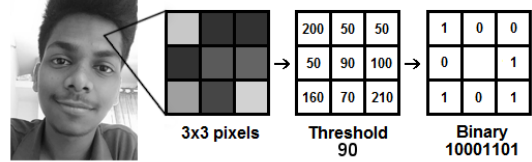

Threshold

Binary
1000110

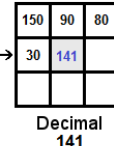

Fig. 5. LBPH Procedure

\subsection{Support Vector Machine}

SVM is a linear model which is used to solve analysis and regression problems. Linear and Non-linear and many practical problems are easily solved by this problem. The patterns are formed by analyzing the large dataset. SVM procedure:

(1) Parallel partition generates two parallel lines which are generated with the help of SVM based on all attributes.

(2) Flat and linear partition is generated by separating the spaces in a single pass.

(3) The hyperplane is used to divide 2 categories by a clear gap.

(4) The data is separated into classes with the help of hyperplane with a large margin.

The closest data points of the classes are represented with the help of the margin of those classes. Figure 6 shows the procedure:

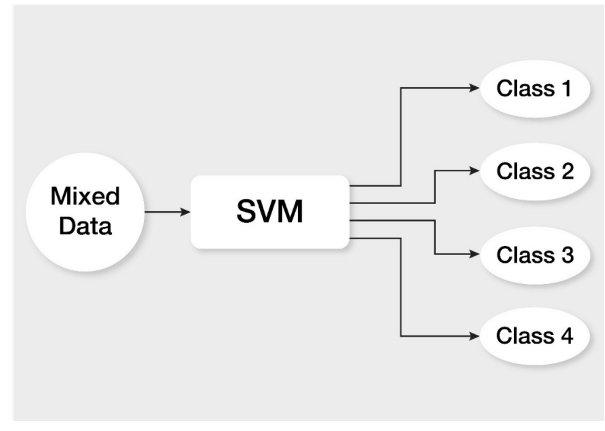

Fig. 6. SVM Procedure

\subsection{QR Code}

A QR code can be read by an imaging device, like a camera. ReedSolomon error correction software is used to format the QR code algorithmically until the point where the picture can be rendered properly [19]. Data is obtained from models which are present in both horizontal and vertical components of the image. The black modules (square dots) which are in the code are designed in a square grid on a white background.

In the proposed system, the QR Code authentication plays an important role of handling the second level security of the system. When a particular student is attempting to mark attendance, his or her face will be classified using ISVM and recognized by the system, the next task of the student is to display the respective QR code in front of the camera to mark his or her attendance.

\section{RESULTS}

After the video, which is recorded, is provided to the system as an input, the system extracts the face from the video and matches with the face which is stored in the database. Once the face is matched, then the QR code is scanned for that particular student to mark the attendance into the database.

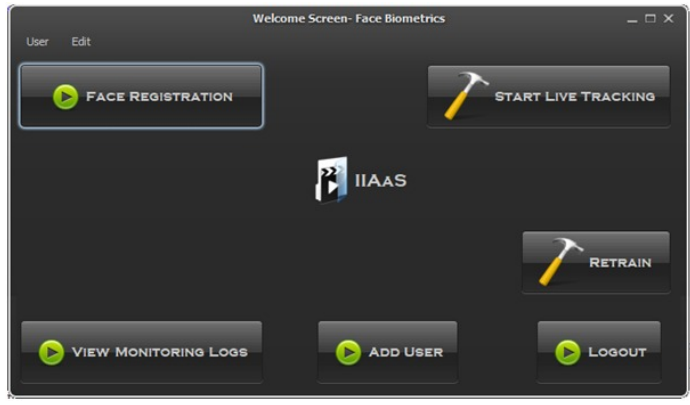

Fig. 7. Basic UI

Figure 7 shows the basic UI of the system which contains face recognition, live tracking, training, registration etc.

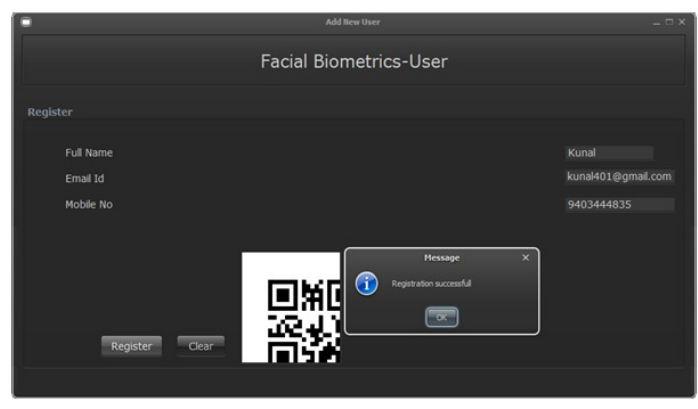

Fig. 8. QR code Registration

Figure 8 shows the registration of the QR code of the particular student in the database.

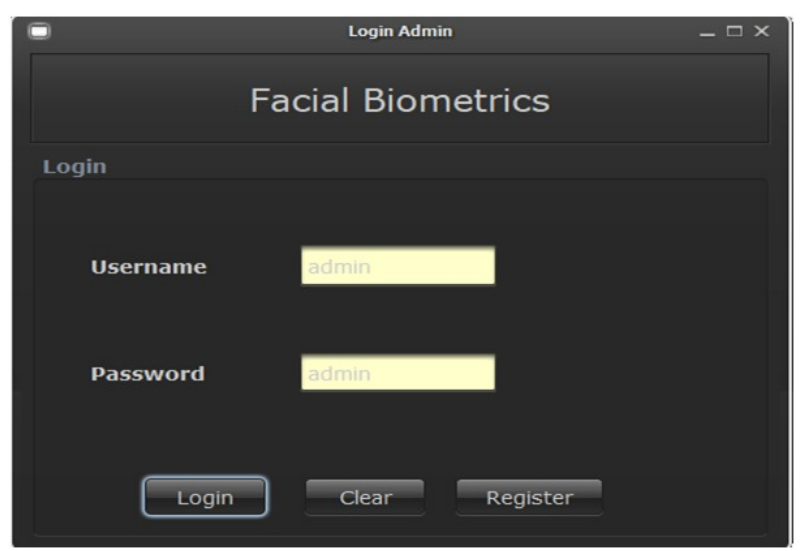

Fig. 9. Login UI

Figure 9 shows the basic UI of the login page where the students can login and see their attendance. 


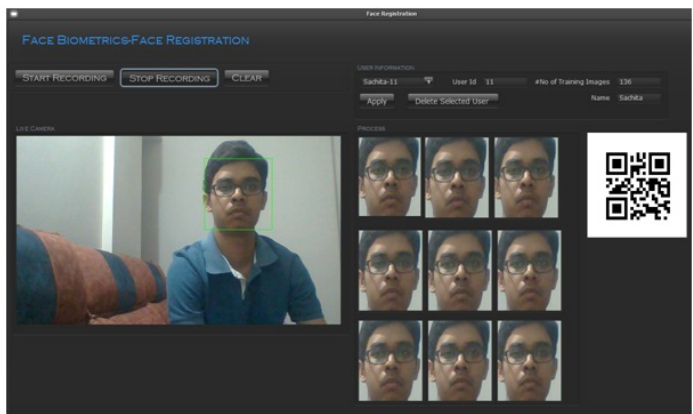

Fig. 10. Face Recognition

Figure 10 shows the live tracking system where the faces are captured from the video.

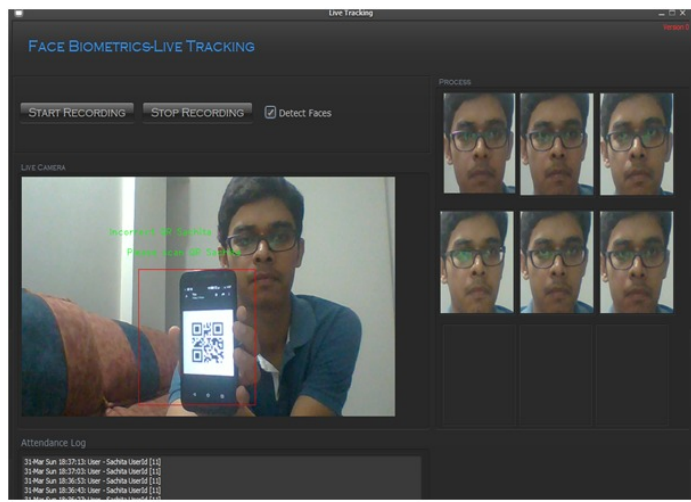

Fig. 11. QR code Scanning

Figure 11 shows the QR code scanning process of the captured face.

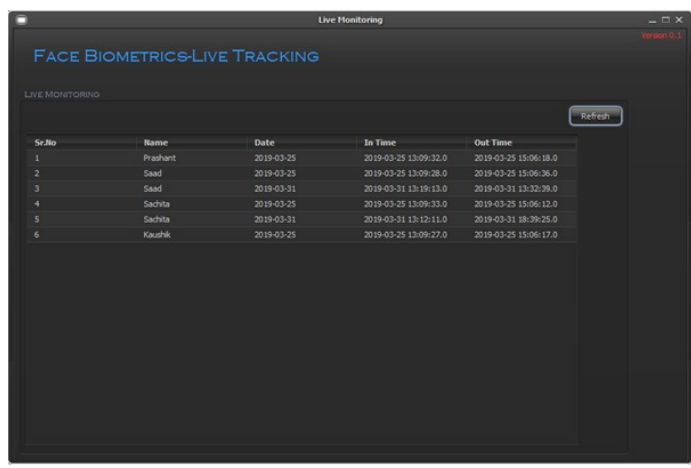

Fig. 12. Database

Figure 12 shows the database where the student's attendance is marked.

$\mathrm{C} 1, \mathrm{C} 2, \mathrm{C} 3, \mathrm{C} 4$ are 4 random classes which are taken into consideration to calculate the classification report of the system. In the second row of Figure 13 , while testing C1, 90 times the class was

\begin{tabular}{|l|r|r|r|r|r|}
\hline & C1 & C2 & C3 & C4 \\
\hline C1 & 161 & 0 & 0 & 0 \\
\hline C2 & 90 & 193 & 0 & 0 \\
\hline C3 & 0 & 0 & 195 & 0 \\
\hline C4 & 0 & 0 & 0 & 206 \\
\hline
\end{tabular}

Fig. 13. Class-wise support distribution

\begin{tabular}{|r|r|r|r|r|}
\hline Sr.No & precision & recall & f1-score & support \\
\hline 1 & 0.64 & 1 & 0.78 & 161 \\
\hline 2 & 1 & 0.68 & 0.81 & 283 \\
\hline 3 & 1 & 1 & 1 & 195 \\
\hline 4 & 1 & 1 & 1 & 206 \\
\hline & & & & \\
\hline avg/total & 0.93 & 0.89 & 0.9 & 845 \\
\hline
\end{tabular}

Fig. 14. Classification report

incorrectly recognized as $\mathrm{C} 2$. Rest all the support distribution is correctly recognized.

Hence, it is evident from Figure 14 that the average precision of the four classes is 0.93 along with an 11 -score of 0.9 which is an exceedingly good result for recognizing true positive rate.

\section{F1-SCORE CLASSWISE}
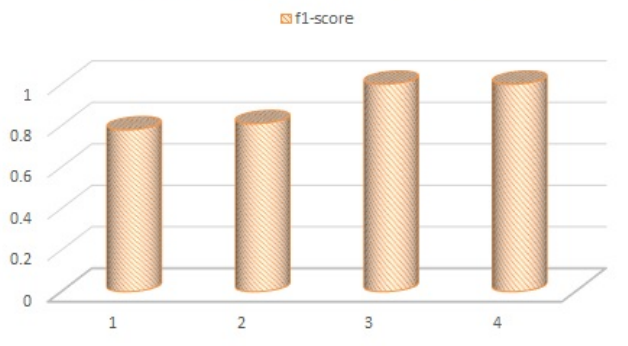

Fig. 15. f1 score Class-wise Classification

The class-wise f1-score of only $\mathrm{C} 1$ and $\mathrm{C} 2$ is near 0.8 as seen in Figure 15, rest of the two classes have an f1-score of 1, which means that the system has the capability to detect real threats and contain low false positives and negatives. Even though f1-score is not comprehended easily, it is safe to say that the system is safe from both false positives and false negatives. Also, f1-score is more useful than accuracy when the class is unevenly distributed.

\section{FUTURE SCOPE}

In future, the application can also be extended in the usage of criminal detection at various places by using the suspected faces in the training database. It can also be used in MNC?s and small-scale startup companies for face detection. By extending or alternating its features, it can also be used for traffic rule violation purpose in place of the traditional CCTV cameras. As far as the time taken to train the images into the system is concerned, it can be improved. 


\section{CONCLUSIONS}

In our previous work [20], various recent methods used by researchers for face detection and recognition along with different orthodox attendance marking techniques were discussed. In this paper, an intuitive system is successfully developed for educational institutions that can be used for monitoring student's attendance in a lecture by detecting and further recognizing the faces of the student's. Taking into consideration the unorthodox algorithms along with the dual security measure using QR Code authentication, the proposed system stands out different from other existing systems. Being intuitive and automated, the system is user-friendly and admin-friendly too. The system gave an average precision rate of 0.93 with $\mathrm{f} 1$ score of 0.9 .

\section{Acknowledgement}

The authors Prashant Wakchaure, Saad Shaikh, Sachita Singh and Kaushik Rohida, would like to thank Prof. Ashish Ramdasi, Project Guide, Dept. of Computer Engineering along with Prof. B.B. Gite, Head of the Department of Computer Engineering at Sinhgad Academy of Engineering, Kondhwa, Pune and anonymous referees, whose help and technical ideas have improved the quality of the paper.

\section{REFERENCES}

[1] Hamdi Dibeklio?lu, Fares Alnajar, Albert Ali Salah, Theo Gevers, Combining Facial Dynamics With Appearance for Age Estimation, IEEE Transactions on Image Processing, Volume: 24, Issue: 6, 1928 - 1943 (2015). doi:10.1109/TIP.2015.2412377.

[2] Yang Zhong, Josephine Sullivan, Haibo Li, Face attribute prediction using off-the-shelf $\mathrm{CNN}$ features, $\mathrm{Pa}$ per presented at the 2016 International Conference on Biometrics (ICB), Halmstad, Sweden, 1 - 7 (2016). doi:10.1109/ICB.2016.7550092.

[3] Max Ehrlich, Timothy J. Shields, Timur Almaev, Mohamed R. Amer, Facial Attributes Classification Using Multi-task Representation Learning, Paper presented at the 2016 IEEE Conference on Computer Vision and Pattern Recognition Workshops (CVPRW),Las Vegas, NV, USA, 752 - 760 (2016). doi:10.1109/CVPRW.2016.99.

[4] Jianlong Fu, Heliang Zheng, Tao Mei, Look Closer to See Better: Recurrent Attention Convolutional Neural Network for Fine-Grained Image Recognition, Paper presented at the 2017 IEEE Conference on Computer Vision and Pattern Recognition (CVPR),Honolulu, HI, USA, 4476 - 4484 (2017). doi:10.1109/CVPR.2017.476.

[5] Yun-Fu Liu, Jing-Ming Guo, Po-Hsien Liu, Jiann-Der Lee, Chen-Chieh Yao, Panoramic Face Recognition, IEEE Transactions on Circuits and Systems for Video Technology, Volume: 28 , Issue: 8, 1864 - 1874 (2017). doi:10.1109/TCSVT.2017.2693682.

[6] Xiang-Yu Li, Zhen-Xian Lin, Face Recognition Based on HOG and Fast PCA Algorithm. In: Krmer P., Alba E., Pan JS., Sn?el V. (eds) Proceedings of the Fourth Euro-China Conference on Intelligent Data Analysis and Applications. ECC 2017. Advances in Intelligent Systems and Computing, vol 682. Springer, Cham, 10-21 (2017). doi:10.1007/978-3-31968527-4_2.
[7] Mashhood Sajid, Rubab Hussain, Muhammad Usman, A conceptual model for automated attendance marking system using facial recognition, Paper presented at the Ninth International Conference on Digital Information Management (ICDIM 2014), Phitsanulok, Thailand 7-10 (2014). doi:10.1109/ICDIM.2014.6991407.

[8] S Poornima, N Sripriya, B Vijayalakshmi, P Vishnupriya, Attendance monitoring system using facial recognition with audio output and gender classification, Paper presented at the 2017 International Conference on Computer Communication and Signal Processing (ICCCSP), Chennai, India, 1-5 (2017). doi:10.1109/ICCCSP.2017.7944103.

[9] Hemantkumar Rathod, Yudhisthir Ware, Snehal Sane, Suresh Raulo, Vishal Pakhare, Imdad A. Rizvi, Automated attendance system using machine learning approach, Paper presented at the 2017 International Conference on Nascent Technologies in Engineering (ICNTE), Navi Mumbai, India, 1-5 (2017). doi:10.1109/ICNTE.2017.7947889.

[10] Yueqi Duan, Jiwen Lu, Jianjiang Feng, Jie Zhou, ContextAware Local Binary Feature Learning for Face Recognition, IEEE Transactions on Pattern Analysis and Machine Intelligence, Volume: 40 ,Issue: 5, 1139 - 1153 (2018). doi:10.1109/TPAMI.2017.2710183.

[11] Samuel Lukas, Aditya Rama Mitra, Ririn Ikana Desanti, Dion Krisnadi, Student attendance system in classroom using face recognition technique, Paper presented at the 2016 International Conference on Information and Communication Technology Convergence (ICTC), Jeju, South Korea, 1032 - 1035 (2016). doi:10.1109/ICTC.2016.7763360.

[12] Manop Phankokkruad, Phichaya Jaturawat, Influence of facial expression and viewpoint variations on face recognition accuracy by different face recognition algorithms, 2017 18th IEEE/ACIS International Conference on Software Engineering, Artificial Intelligence, Paper presented at the 2017 18th IEEE/ACIS International Conference on Software Engineering, Artificial Intelligence, Networking and Parallel/Distributed Computing (SNPD), Kanazawa, Japan, 231 237 (2017). doi:10.1109/SNPD.2017.8022727.

[13] Hamdi Dibeklio?lu, Albert Ali Salah, Theo Gevers, Are You Really Smiling at Me? Spontaneous versus Posed Enjoyment Smiles. In: Fitzgibbon A., Lazebnik S., Perona P., Sato Y., Schmid C. (eds) Computer Vision ? ECCV 2012. ECCV 2012. Lecture Notes in Computer Science, vol 7574. Springer, Berlin, Heidelberg, 525-538 (2012). doi:10.1007/978-3-64233712-3_38.

[14] Ziwei Liu, Ping Luo, Xiaogang Wang, Xiaoou Tang, Deep Learning Face Attributes in the Wild, Paper presented at the 2015 IEEE International Conference on Computer Vision (ICCV), Santiago, Chile, 3730 - 3738 (2015). doi:10.1109/ICCV.2015.425.

[15] Shao-Dong Lv, Yong-Duan Song, Mei Xu, Cong-Ying Huang, Face Detection under Complex Background and Illumination, Journal of Electronic Science and Technology, 7882 (2015). doi:10.3969/j.issn.1674-862X.2015.01.014.

[16] Raghav Apoorv, Puja Mathur, Smart attendance management using Bluetooth Low Energy and Android, Paper presented at the 2016 IEEE Region 10 Conference (TENCON), Singapore, 1048 - 1052 (2016). doi:10.1109/TENCON.2016.7848166.

[17] M. K. Yeop, M. Z. A. Abdul Aziz, M. S. R. Mohd Shah, M. F. Abd Kadir, Smart Attendance System by using RFID, 
Paper presented at the 2007 Asia-Pacific Conference on Applied Electromagnetics, Melaka, Malaysia, 1 - 4 (2007). doi:10.1109/APACE.2007.4603906.

[18] M.A. Meor Said, M.H. Misran, M.A. Othman, M.M. Ismail, H.A. Sulaiman, A. Salleh, N. Yusop, Biometric attendance, Paper presented at the 2014 International Symposium on Technology Management and Emerging Technologies, Bandung, Indonesia, 258 - 263 (2014). doi:10.1109/ISTMET.2014.6936516.

[19] Fadi Masalha, Nael Hirzallah, A Students Attendance System Using QR Code, (IJACSA) International Journal of Advanced Computer Science and Applications, Vol. 5, No. 3, 75 - 79 (2014). doi:10.14569/issn.2156-5570.

[20] Prashant Wakchaure, Saad Shaikh, Sachita Singh, Kaushik Rohida, Prof Ashish Ramdasi, Literature Survey - Improved Intuitive Automated Attendance System Using Unorthodox Algorithms, INTERNATIONAL JOURNAL OF RESEARCH IN ELECTRONICS AND COMPUTER ENGINEERING (IJRECE), Vol.6, Issue: 4 (Version 5), 1480 - 1484 (2018). http://nebula. wsimg.com/911f286adcdcfe741d85aac5707541ca? AccessKeyId=DFB1BA3CED7E7997D5B1\&disposition= 0\&alloworigin=1 